\title{
Activity induced synchronization: Mutual flocking and chiral self-sorting
}

\author{
D. Levis, ${ }^{1,2,3,{ }^{*}}$ I. Pagonabarraga, ${ }^{1,2,3}$ and B. Liebchen ${ }^{4,5,6, \dagger}$ \\ ${ }^{1}$ CECAM Centre Européen de Calcul Atomique et Moléculaire, École Polytechnique Fédérale de Lausanne, \\ Batochime, Avenue Forel 2, 1015 Lausanne, Switzerland \\ ${ }^{2}$ Departament de Física de la Matèria Condensada, Universitat de Barcelona, Martí i Franquès 1, E08028 Barcelona, Spain \\ ${ }^{3}$ UBICS University of Barcelona Institute of Complex Systems, Martí i Franquès 1, E08028 Barcelona, Spain \\ ${ }^{4}$ SUPA, School of Physics and Astronomy, University of Edinburgh, Edinburgh EH9 3FD, United Kingdom \\ ${ }^{5}$ Institut für Theoretische Physik II: Weiche Materie, Heinrich-Heine-Universität Düsseldorf, D-40225 Düsseldorf, Germany \\ ${ }^{6}$ Institut für Festkörperphysik, Technische Universität Darmstadt, D-64289 Darmstadt, Germany
}

(Received 5 September 2018; published 24 September 2019)

\begin{abstract}
Synchronization, the temporal coordination of coupled oscillators, allows fireflies to flash in unison, neurons to fire collectively, and human crowds to fall in step on the London millenium bridge. Here, we interpret active (or self-propelled) chiral microswimmers with a distribution of intrinsic frequencies as motile oscillators and show that they can synchronize over very large distances, even for local coupling in two dimensions (2D). This opposes canonical nonactive oscillators on static or time-dependent networks, leading to synchronized domains only. A consequence of this activity-induced synchronization is the emergence of a "mutual flocking phase," where particles of opposite chirality cooperate to form superimposed flocks moving at a relative angle to each other, providing a chiral active matter analogue to the celebrated Toner-Tu phase. The underlying mechanism employs a positive feedback loop involving the two-way coupling between oscillators' phase and self-propulsion and could be exploited as a design principle for synthetic active materials and chiral self-sorting techniques.
\end{abstract}

DOI: 10.1103/PhysRevResearch.1.023026

\section{INTRODUCTION}

Populations of motile entities, from bacteria to synthetic microswimmers, can spontaneously self-organize into phases which are unattainable in equilibrium passive matter. Examples range from the spectacular murmuration of starlings and bacterial swarming [1-4], to collective behavior of synthetic self-propelled grains [5,6], emulsion droplets [7], and assemblies of robots [8]. Active colloids [9], in particular, spontaneously form living clusters in low-density suspensions which, unlike equilibrium clusters, continuously break up and reform [9-15]. Remarkably, when active particles align with their neighbors, they can self-organize into a polarly ordered phase, featuring long-range order in two dimensions (2D) [16-21]. Thus, motile entities, like wildebeests or sheep, can order at long distances and all run in the same direction, whereas immotile entities like spins in a 2D magnet cannot display (true) long-range order when locally coupled [22,23]. Likewise, oscillators which are localized in space, like metronomes or neurons [24,25], can synchronize only locally in 2D or three dimensions (3D) when coupled to their neighbors [26-28].

\footnotetext{
*Corresponding author: demian.levis@epfl.ch

${ }^{\dagger}$ Corresponding author: liebchen@fkp.tu-darmstadt.de
}

Published by the American Physical Society under the terms of the Creative Commons Attribution 4.0 International license. Further distribution of this work must maintain attribution to the author(s) and the published article's title, journal citation, and DOI.
Here, we consider ensembles of chiral active particles, such as certain microorganisms [29-33], cell components [34-36], and synthetic microswimmers [37-44]. We interpret them as active oscillators and ask for their large-scale and long-time synchronization behavior: (i) In active matter, previous studies on chiral active particles have focused on pattern formation [44-49] but leave the synchronization behavior (frequency locking) of large ensembles essentially open, irrespective of previous work focusing on phase locking in monofrequent ensembles [50] and on similarities between the Kuramoto and Vicsek models [51]. Notice that the understanding of the large-scale synchronization in generic chiral active particles with a frequency distribution is a fundamentally different problem than the emergence of long-range order in a system of identical active particles as studied, e.g., in the Vicsek and the Toner-Tu models. To see this, consider that for "passive oscillators" (Kuramoto model with local coupling), the lower critical dimension $D_{c}=2$ for the emergence of synchronization but $D_{c}=4$ for oscillators with a frequency distribution $D_{c}=4$ [26-28]. Therefore, while we know that identical chiral active particles can synchronize over long distances in $2 \mathrm{D}[16,17,44]$, it is fully unclear if generic ensembles can synchronize in fewer than four dimensions. (ii) In the field of synchronization, in turn, much is known on the largescale synchronization of oscillators which are localized in space $[24,25,52]$ or move in a way that is unaffected by their phases [53-56] - but not for active oscillators showing a two-way coupling between phase and displacement through space.

Here, we show that generic active oscillators featuring a frequency distribution can synchronize over large distances 

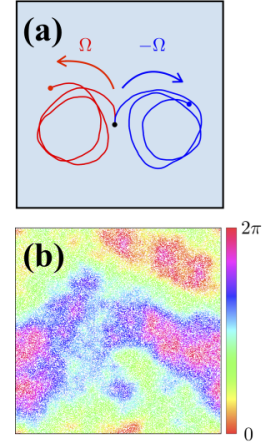

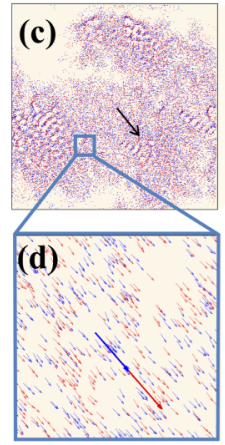

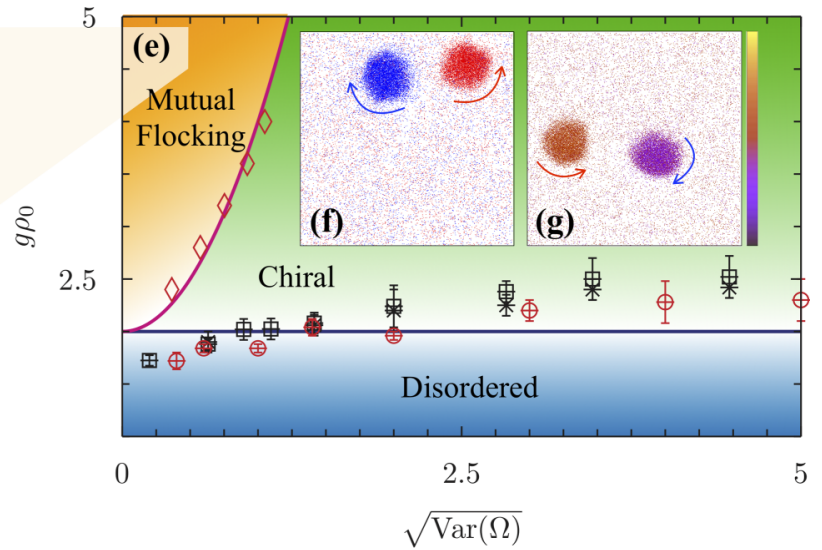

FIG. 1. (a) Trajectories of particles with opposite chirality. (b) Late-time snapshot of $N=10^{5}$ mobile Kuramoto oscillators with a Gaussian frequency distribution, after relaxation from a completely synchronized state $\left(\operatorname{Var}(\Omega)=0.4\right.$ and $\left.g \rho_{0}=2.8\right)$. Colors represent oscillator phases. The dispersion of natural frequency spontaneously destabilizes the initially ordered state. (c) Mutual flocking: $N=3 \times 10^{4}$ chiral active particles with $\Omega= \pm 0.6$ and $g \rho_{0}=40$ forming a globally polarized and synchronized phase; panel (d) shows a detailed view. Small arrows show particle orientations; large ones show the average polarization direction of both species represented in red and blue (arrow lengths are arbitrary). (e) Phase diagram of active oscillators. Symbols show simulation results for the phase boundaries (red symbols for two species, black ones for a normal distribution) using two different densities ( $\rho_{0}=10,20$ in black crosses and squares); lines show analytical predictions $\left(g \rho_{0}=2[1+\operatorname{Var}(\Omega)]\right.$ and $\left.g \rho_{0}=2\right)$. The weak deviation between simulations and the $g \rho_{0}=2$ line comes from a reduction of the growth rate of unstable modes as $\Omega$ increases (see the Supplemental Material [57]). Insets show snapshots in the chiral phase for two species (f) and a Gaussian distribution $(\mathrm{g})$ both for $\operatorname{Var}(\Omega)=2 ; g \rho_{0}=2.8$; colors show intrinsic frequencies.

even in 2D. We call this phenomenon "activity-induced synchronization" and note that it can by no means be concluded directly from the well-known fact that identical active particles can show long-range order $[16,17]$ and synchronize in $2 \mathrm{D}$ [44]. Instead, it appears surprising since generic ensembles of static oscillators with a frequency distribution can synchronize only locally in 2D and in 2D [26-28]. The mechanism creating activity-induced synchronization is rooted in the two-way coupling between phase and direction of motion of the oscillators. This key ingredient in our model, which is naturally present in chiral active particles, triggers a positive feedback loop between synchronization and coordinated oscillator motion, enhancing the interaction time of the active oscillators. This feedback determines the phase diagram of generic active oscillators, leading to phases having no counterpart in either identical active oscillators [44] or in immotile oscillators: For comparatively small frequency distributions, active oscillators self-organize into a mutual flocking phase. Here, active oscillators with opposite chirality cooperate to move coherently at a relative angle, forming two nonrotating superimposed flocks mutually stabilizing each other. This phase is akin to the celebrated Toner-Tu phase in linear active matter [17-19] [see Fig. 1(e), orange domain, and the snapshots Figs. 1(c) and 1(d)]. For ensembles with a comparatively large frequency dispersion, the active oscillators spatially segregate according to their chirality and form rotating macroscopic clusters (see Fig. 1, green domain), featuring internal synchronization [see snapshots Figs. 1(f) and 1(g)] and growing linearly with system size. This chiral phase constitutes a second example where activity leads to synchronization on the macroscale. Notice that the chiral self-sorting underlying this phase allows active particles to segregate by chirality, without requiring chemical reactions [58], external flows, or environmental chirality [40].
To demonstrate the impact of the feedback between phase and displacement on the large-scale synchronization of coupled oscillators, we consider $N$ overdamped particles with 2D positions $\boldsymbol{r}_{\alpha}$ and alignment interactions of strength $K$ in a square box of length $L$, which self-propel with a constant speed $v$ along direction $\boldsymbol{n}_{\alpha}=\left(\cos \theta_{\alpha}, \sin \theta_{\alpha}\right)$, as described by

$$
\begin{gathered}
\dot{\boldsymbol{r}}_{\alpha}=v \boldsymbol{n}_{\alpha}, \\
\dot{\theta}_{\alpha}=\omega_{\alpha}+\frac{K}{\pi R^{2}} \sum_{\nu \in \partial_{\alpha}} \sin \left(\theta_{\nu}-\theta_{\alpha}\right)+\sqrt{2 D_{r}} \eta_{\alpha} .
\end{gathered}
$$

Here, $\eta$ represents Gaussian white noise with unit variance and zero mean. The intrinsic frequencies $\omega_{\alpha}$ are randomly drawn from a distribution $\Delta(\omega)$. Note that, unlike more complicated models, involving, e.g., hydrodynamic interactions, excluded volume effects, or sophisticated couplings, the present model focuses on the essential ingredients required to demonstrate the generic synchronization scenario shown by active oscillators. Moreover, direct experimental realizations of the present model are also possible, e.g., using 3D-printed granular particles on vibrated plates $[6,59,60]$. For example, disk-shaped granulates featuring asymmetric legs [6] both swim in circles and locally align with each other [6,59]. References $[35,61,62]$ provide other very recent setups containing the key ingredients of the present model. Since we want to understand the synchronization behavior arising from the competition between the distribution of natural frequencies and the coupling for chiral active particles, we consider only distributions with zero mean. Indeed, here we establish the role played by the frequency dispersion [through its variance $\operatorname{Var}(\omega)$ ] on the collective behavior of active oscillators. The sum of Eq. (2) runs over all the neighbors of particle $\alpha$, defined by the cutoff distance $R$. For $v=0$, our model reduces 


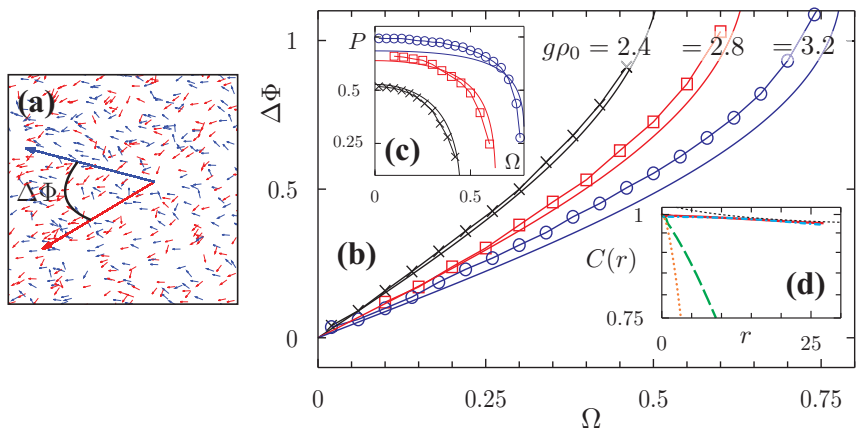

FIG. 2. Mutual flocking phase. Detailed view of a late-time configuration for $\Omega= \pm 1.5$ at $g \rho_{0}=5.6$ (a). Thick arrows show the average polarization of both species, while small arrows show particle orientations. Angle $\Delta \Phi(|\Omega|)$ between the two flocks (b) and partial polarization $P(|\Omega|)$ (c) of each species. Lines show Eq. (5) and points simulation results for $\mathrm{Pe}=2 ; \rho_{0}=200$. (d) $C(r$ ) (in linear-log scale) of a system of active oscillators $(\mathrm{Pe}=2$, plain red line), static oscillators (in green), Brownian oscillators (in orange), and underdamped velocity-aligning oscillators (in blue) (in all cases $\operatorname{Var}(\Omega)=0.4$ and $g \rho_{0}=40$ ) (see Ref. [57] for details). The dotted black line shows an exponential decay to the asymptotic value $P^{2}$.

to the noisy Kuramoto model of locally coupled oscillators, which is known to show only local synchronization in 2D $[27,28]$. For $\omega_{\alpha}=0$, in turn, the model is equivalent to (a smooth variant of) the Viscek model [63,64] for which it is known that self-propulsion induces long-range order in 2D. The key feature of this model is that it identifies the oscillators' phase with their direction of self-propulsion. In the spirit of the Kuramoto model [65] and simple models of self-propelled particles (like the Vicsek and smooth variants of it $[63,64,66]$ or the active Brownian particle model [67-69]), the model provides a minimal framework to study the generic behavior emerging in systems of nonidentical active oscillators. We demonstrate the generality of our results by considering a multifrequent extension of the underdamped, velocity-aligning (but not self-propelled) particles introduced in Ref. [50]. These nonequilibrium particles feature the same two-way coupling between orientation (phase) and displacement and show an analogous synchronization behavior as we obtain for chiral active particles (see Fig. 2(d) and Ref. [57]).

Notice that most previous studies on synchronization in dynamic networks have focused on identical oscillators whose phase does not affect their motion in space [53-56], showing that the absence of global synchronization (and other scaling properties) for Kuramoto oscillators in 2D is generically preserved, even if carried by active particles. Note that despite this recent boost of activity on synchronization of motile oscillators, the dispersion of natural frequencies, a main feature in synchronization problems, has not been explored in detail. Therefore, to better understand the fundamental synchronization mechanisms involved in active systems, we have carried extensive simulations of our model in the absence of the back-coupling between the phase of the oscillators and their direction of motion-i.e., a model of nonidentical oscillators attached to self-propelled particles for which $\boldsymbol{n}_{\alpha}$ performs a free diffusion (see the Supplemental Material [SM] [57] for further details). As illustrated in Fig. 1(b) and shown in more detail in the SM [57], global phase synchronization cannot be achieved in the absence of the back-coupling between phase and motility. Models considering agents whose phase, or a different internal state, affect the way they move in space appeared recently [70-74] but did not consider particles self-propelling in the direction of their phase (with a distribution of natural frequencies and local coupling), which is the key ingredient of the present model. To understand the key control parameters of the present system, we express times and lengths in units of $1 / D_{r}$ and $R$, respectively, leading to the dimensionless quantities: (i) $g=K /\left(\pi R^{2} D_{r}\right)$, the reduced coupling; (ii) $\mathrm{Pe}=v /\left(D_{r} R\right)$, the rotational Péclet number; (iii) $\Omega_{i}=\omega_{i} / D_{r}$, the reduced rotation frequency; and (iv) $\rho_{0}=N R^{2} / L^{2}$, the average number density. We denote by $\rho^{(i)}=N^{(i)} R^{2} / L^{2}$ the density of particles with natural frequency $\Omega_{i}$ (species $i$ ), where $N^{(i)}$ counts particles sharing the same natural frequency. For simplicity, we exemplify our key results for two species with equal overall density and opposite chirality. As we will show, the synchronization behavior is controlled by $\operatorname{Var}(\Omega)$, such that this distribution is largely representative of cases of several species and continuous frequency distributions.

\section{HYDRODYNAMIC THEORY}

We first derive a set of field equations, describing the collective dynamics of chiral active particles, mainly following Refs. [64,75-77]. Here, we assume that the system contains $M$ different species with frequencies $\Omega_{i}(i=1 \ldots M)$ and describe the dynamics of species $i$ particles using the density field $\rho \equiv \rho^{(i)}(\mathbf{x}, t)$ and the polarization density $\mathbf{w} \equiv$ $\mathbf{w}^{(i)}(\mathbf{x}, t)$, where $\mathbf{w} /|\mathbf{w}|$ is the average self-propulsion direction of particles of species $i$. The resulting equations read (see the SM for details [57]):

$$
\begin{gathered}
\dot{\rho}=-\operatorname{Pe} \nabla \cdot \mathbf{w} \\
\dot{\mathbf{w}}=-\mathbf{w}+\sum_{i=1}^{M} \frac{g \rho}{2} \mathbf{w}^{(i)}+\Omega \mathbf{w}_{\perp}-\frac{\mathrm{Pe}}{2} \nabla \rho \\
+\frac{\mathrm{Pe}^{2}}{2 b} \nabla^{2} \mathbf{w}+\frac{\mathrm{Pe}^{2} \Omega}{4 b} \nabla^{2} \mathbf{w}_{\perp} \\
-\frac{g^{2}}{b}\left(\mathbf{w}+\frac{\Omega \mathbf{w}_{\perp}}{2}\right)\left(\sum_{i=1}^{M} \mathbf{w}^{(i)}\right)^{2}+O\left(\nabla \mathbf{w}^{2}\right)
\end{gathered}
$$

Here, $b=2\left(4+\Omega^{2}\right), \mathbf{w}_{\perp}=\left(-w_{y}, w_{x}\right)$, and $O\left(\nabla \mathbf{w}^{2}\right)$ represent terms which are both nonlinear in $\mathbf{w}$ and involve gradients, and these are not of interest for our purposes. Equation (3) simply reflects that particles on average self-propel in the direction of w. The first term in Eq. (4) represents a decay of the polarization due to rotational diffusion of the particles, which happens in competition with the second term, creating alignment among all species; the third term represents a rotation of the average (local) polarization direction with a species-specific frequency while the fourth term expresses a statistical tendency for self-propulsion away from highdensity regions; remaining terms "smear out" regions of high and low polarization and lead to saturation. For weak interactions, the system is in a disordered uniform phase, given by 
$(\rho, \mathbf{w})=\left(\rho_{0}, \mathbf{0}\right)$, which is a solution of Eqs. (3) and (4) and represented by the blue domain in the phase diagram Fig. 1(e).

\section{MUTUAL FLOCKING PHASE}

To understand the onset of synchronization, we perform a linear stability analysis for a bimodal frequency distribution $\Omega_{1}=-\Omega_{2}=\Omega$ of the uniform disordered phase $\left(\rho^{(1),(2)}=\right.$ $\rho_{0} / 2, \mathbf{w}_{1}=\mathbf{w}_{2}=0$ ) [57]. It is instructive to first focus on the zero wave-number limit $(q=0)$. Here, we find that the uniform phase loses stability if $g \rho_{0}>2\left(1+\Omega^{2}\right)$, suggesting the existence of a nontrivial ordered uniform phase. We indeed find an exact solution of our field equations (3) and (4) representing such a phase: a state of uniform density $\rho^{(1)}=\rho^{(2)}=$ $\rho_{0} / 2$, but with a finite polarization, spontaneously breaking the symmetry of the disordered phase. In this state, circle swimmers of both species cooperate and form two individual and superimposed flocks moving linearly and at a relative angle $\Delta \Phi$ to each other. We thus call it the mutual flocking phase. Defining the overall polarization $P=\left|\mathbf{w}_{1}+\mathbf{w}_{2}\right| / \rho_{0}$, where $\left|\mathbf{w}_{1}\right|=\left|\mathbf{w}_{2}\right|=\rho_{0} P / \sqrt{2(1+\cos \Delta \Phi)}$, the exact expressions representing the mutual flocking phase read

$$
\begin{gathered}
P=\frac{\sqrt{2}}{g \rho_{0}} \sqrt{\sqrt{\left(g \rho_{0}\right)^{2}+6 \Omega^{2}\left(g \rho_{0}-6\right)}+g \rho_{0}+2 \Omega^{2}-4}, \\
\Delta \Phi=-i \ln \left[\frac{2 \Omega\left(6-\frac{g \rho_{0}}{2}\right)+4 i \sqrt{\frac{\left(g \rho_{0}\right)^{2}}{4}+3 \Omega^{2}\left(\frac{g \rho_{0}}{2}-3\right)}}{g \rho_{0}(\Omega+2 i)}\right] .
\end{gathered}
$$

At low frequency, $\Omega<1 / \sqrt{2}$, this solution exists (is real and positive) if $g \rho_{0}>2\left(1+\Omega^{2}\right)$, i.e., precisely when the field equations show a linear instability at $q=0$.

Brownian dynamics simulations at high coupling and high density confirm the existence of the mutual flocking phase. As shown in Fig. 1(e), we find the mutual flocking phase essentially in the whole parameter regime, where it exists according to our field theory. Figure 2 shows in turn a close quantitative agreement between theoretical predictions [Eqs. (5)] and our simulations, both for the angle between the two flocks and for the overall polarization (see movie 1 [57]). (In our simulations, we measure the partial and overall polarization, $\tilde{P}=\left|\mathbf{P}_{1,2}\right|$, where $\mathbf{P}_{i}=\frac{2}{N} \sum_{\alpha} \mathbf{n}_{\alpha} \delta_{\omega_{\alpha}, \omega_{i}}$, and $P=\frac{1}{N}\left|\sum_{\alpha} \mathbf{n}_{\alpha}\right|$, respectively.) In Fig. 2(d), we show the orientational selfcorrelation function $C(r)=\left\langle\boldsymbol{n}_{i} \cdot \boldsymbol{n}_{j}\right\rangle$, strongly suggesting the emergence of global synchronization only for systems of oscillators whose phase directly affects their motion, in contrast to immobile oscillators $[v=0$ in Eq. (1)] or systems of mobile oscillators which move independently of their phase (see Ref. [57]).

\section{ACTIVITY-INDUCED SYNCHRONIZATION}

To understand when the disordered phase loses stability, we now explore its linear stability at finite wave number $q \neq 0$. This is equivalent to accounting for the impact of motility on the onset of synchronization, as the coefficients of all gradient terms in (3) and (4) are nonvanishing only if $\mathrm{Pe} \neq 0$. While static oscillators need a stronger coupling to synchronize as the frequency dispersion increases $[25,65]$, our linear stability
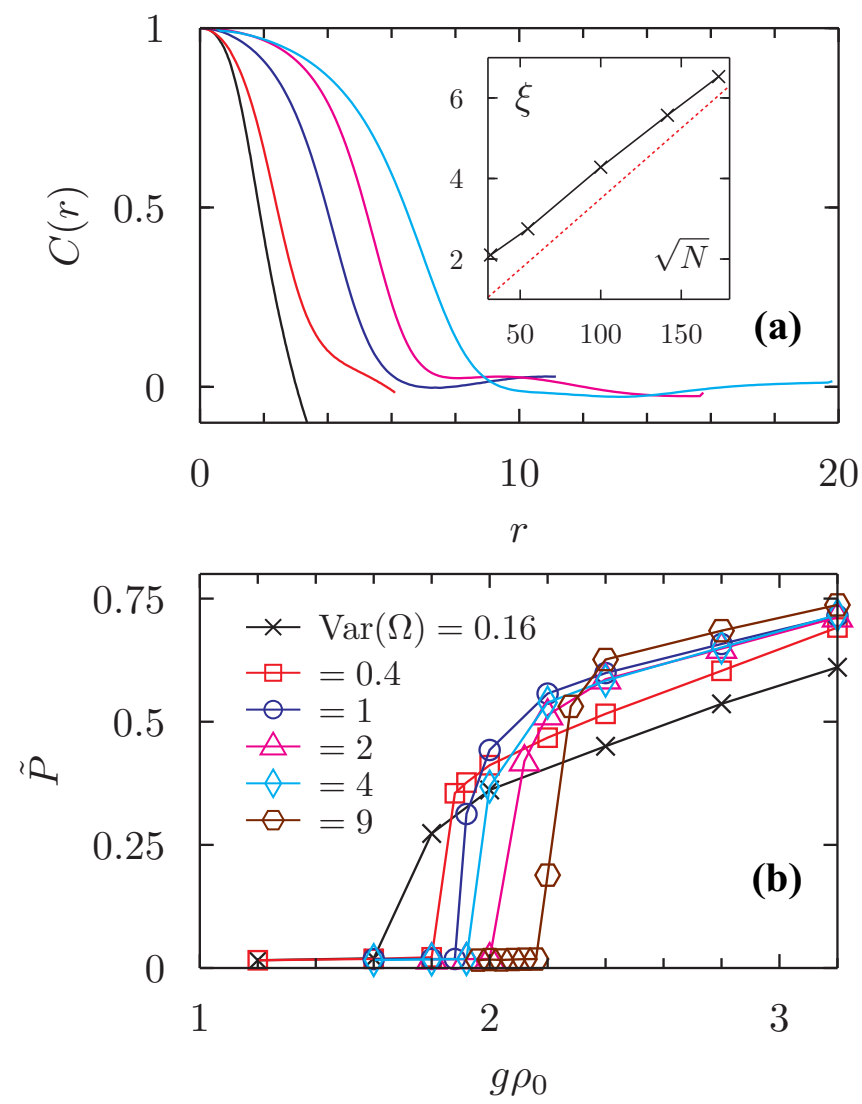

FIG. 3. Chiral phase: (a) spatial correlations for $g \rho_{0}=2.8$, $\operatorname{Var}(\Omega)=2$ and several system sizes $\left(N=10^{3}, \ldots, 3.10^{4}\right.$, from left to right). The inset shows the corresponding correlation length $\xi$ as a function of $\sqrt{N}$. We show for comparison a linear growth $\propto \sqrt{N}$ in dotted line. (b) Partial polarization $\tilde{P}$ as a function of $g \rho_{0}$ for several frequency dispersions.

calculation shows that coupling phase and motility generally induces an instability for any $g \rho_{0}>2$ [57], independently of $\operatorname{Var}(\Omega)$. Activity thus induces phase ordering even in parameter regimes where a corresponding nonmotile system $(\mathrm{Pe}=0)$ would simply show asynchronous oscillations of the individual particles. As the present instability emerges for localized perturbations, we do not expect a uniform phase but rather the emergence of localized synchronous structures. Our particle-based simulations confirm this. Above the critical value $g \rho_{0} \gtrsim 2$ [Fig. 1(e)], we find large rotating clusters of opposite chirality featuring internal phase synchronization, as illustrated by the snapshots in Figs. 1(f) and 1(g) (and movie 2 [57]). From $C(r)$, we extract a characteristic length $\xi$, which, as shown in Fig. 3(a), grows linearly with $\sqrt{N}$ (at fixed $\rho_{0}$ ), meaning that clusters are macroscopic in this regime and tend to phase separate. Thus, the chiral phase represents a second example where activity induces (long-range) phase synchronization among species. As shown in Fig. 3(b), above a critical coupling strength, $\tilde{P}$ starts to increase, allowing us to locate the phase boundaries [Fig. 1(e)]. (Particle segregation, quantified by the probability $\Psi$ to find an excess of particles of one chirality, offers an alternative route to identify the emergence of the chiral phase; see Ref. [57].) 


\section{CONTINUOUS FREQUENCY DISTRIBUTIONS}

To demonstrate the generality of our results, we now simulate active oscillators with Gaussian distribution of natural frequencies [with zero mean and variance $\operatorname{Var}(\Omega)$ ]. As for two species, we predict the stability threshold of the disordered phase at $g \rho_{0}>2$ for three and four species using linear stability analysis (see SM [57]) and confirm with simulations that the stability threshold of the disordered state does not change as compared to the two-species case, up to numerical accuracy [Fig. 1(e)]. At $g \rho_{0} \gtrsim 2$, we find the same two phases as for two species: a mutual flocking phase, further illustrated in the SM [57], and a phase composing synchronized macroclusters scaling linearly with system size (see Fig. $1(\mathrm{~g})$ and movie 3 [57]). Now, the macroclusters involve a continuos range of frequencies and feature frequency synchronization across species.

\section{PHYSICAL MECHANISM}

What is the physical mechanism allowing motility to qualitatively change the synchronization of oscillators? It is well known that oscillator motion itself does not affect the absence of global synchronization in $D<D_{c}=4$ [56,57]. Hence, compared to oscillators on dynamic networks, the crucial ingredient of active oscillators (circle swimmers, Eqs. (1) and (2) and the underdamped, velocity aligning ones [50,57]) is that their phase influences their direction of motion. It follows that two circle swimmers sharing the same phase are aligned and move together, enhancing their interaction time, which in turn enhances their alignment and fosters synchronization. This can be viewed as a positive feedback between alignment (local synchronization) and interaction time, mutually supporting each other, which is absent for oscillators on dynamic networks but plays a fundamental role for synchronization of active oscillators. The impact of such feedback on the emergence of order is even more dramatic than in linear active matter: The critical dimension for the occurrence of global phase synchronization seems to change from $D_{c}=4$ (passive oscillators) to $D_{c}=2$ for active oscillators.

\section{CONCLUSIONS}

Chiral active particles, here interpreted as 2D active oscillators, can synchronize over very long distances, even for a purely local coupling. This contrasts the synchronization behavior of the huge class of nonactive oscillators in $2 \mathrm{D}$ or $3 \mathrm{D}$ structures [25], which can only synchronize within domains. A consequence of global synchronization in active oscillators is the emergence of the mutual flocking phase as a new active matter phase, akin to the celebrated Toner-Tu phase in linear active matter. Our results transcend a knowledge boundary at the interface of active matter physics and synchronization and could be useful, e.g., to sort active enantiomers.

\section{ACKNOWLEDGMENTS}

The authors thank H. Löwen and D. Marenduzzo for fruitful discussions. D.L. and B.L. acknowledge received funding from a Marie Curie Intra European Fellowship (Grant Agreement No. 654908 and Grant Agreement No. 657517) within Horizon 2020. I.P. acknowledges MINECO and DURSI for financial support under Projects No. FIS2015-67837-P and No. 2017SGR-884, respectively.

D.L. and B.L. contributed equally to this work.
[1] D. J. T. Sumpter, Collective Animal Behavior (Princeton University Press, Princeton, NJ, 2010).

[2] H.-P. Zhang, A. Beer, E.-L. Florin, and H. L. Swinney, Collective motion and density fluctuations in bacterial colonies, Proc. Nat. Acad. Sci. USA 107, 13626 (2010).

[3] T. Vicsek and A. Zafeiris, Collective motion, Phys. Rep. 517, 71 (2012).

[4] M. C. Marchetti, J.-F. Joanny, S. Ramaswamy, T. B. Liverpool, J. Prost, M. Rao, and R. A. Simha, Hydrodynamics of soft active matter, Rev. Mod. Phys. 85, 1143 (2013).

[5] V. Narayan, S. Ramaswamy, and N. Menon, Long-lived giant number fluctuations in a swarming granular nematic, Science 317, 105 (2007).

[6] J. Deseigne, O. Dauchot, and H. Chaté, Collective Motion of Vibrated Polar Disks, Phys. Rev. Lett. 105, 098001 (2010).

[7] T. Sanchez, D. T. N. Chen, S. J. DeCamp, M. Heymann, and Z. Dogic, Spontaneous motion in hierarchically assembled active matter, Nature (London) 491, 431 (2012).

[8] M. Rubenstein, A. Cornejo, and R. Nagpal, Programmable self-assembly in a thousand-robot swarm, Science 345, 795 (2014)

[9] C. Bechinger, R. Di Leonardo, H. Löwen, C. Reichhardt, G. Volpe, and G. Volpe, Active particles in complex and crowded environments, Rev. Mod. Phys. 88, 045006 (2016).
[10] I. Theurkauff, C. Cottin-Bizonne, J. Palacci, C. Ybert, and L. Bocquet, Dynamic Clustering in Active Colloidal Suspensions with Chemical Signaling, Phys. Rev. Lett. 108, 268303 (2012).

[11] J. Palacci, S. Sacanna, A. P. Steinberg, D. J. Pine, and P. M. Chaikin, Living crystals of light-activated colloidal surfers, Science 339, 936 (2013).

[12] D. Levis and L. Berthier, Clustering and heterogeneous dynamics in a kinetic Monte Carlo model of self-propelled hard disks, Phys. Rev. E 89, 062301 (2014).

[13] R. Soto and R. Golestanian, Run-and-tumble dynamics in a crowded environment: Persistent exclusion process for swimmers, Phys. Rev. E 89, 012706 (2014).

[14] B. Liebchen, D. Marenduzzo, and M. E. Cates, Phoretic Interactions Generically Induce Dynamic Clusters and Wave Patterns in Active Colloids, Phys. Rev. Lett. 118, 268001 (2017).

[15] F. Ginot, I. Theurkauff, F. Detcheverry, C. Ybert, and C. CottinBizonne, Aggregation-fragmentation and individual dynamics of active clusters, Nat. Commun. 9, 696 (2018).

[16] T. Vicsek, A. Czirók, E. Ben-Jacob, I. Cohen, and O. Shochet, Novel Type of Phase Transition in a System of Self-Driven Particles, Phys. Rev. Lett. 75, 1226 (1995).

[17] J. Toner and Y. Tu, Long-Range Order in a Two-Dimensional Dynamical $x y$ Model: How Birds Fly Together, Phys. Rev. Lett. 75, 4326 (1995). 
[18] J. Toner and Y. Tu, Flocks, herds, and schools: A quantitative theory of flocking, Phys. Rev. E 58, 4828 (1998).

[19] J. Toner, Y. Tu, and S. Ramaswamy, Hydrodynamics and phases of flocks, Ann. Phys. 318, 170 (2005).

[20] F. Ginelli, F. Peruani, M. Bär, and H. Chaté, Large-Scale Collective Properties of Self-Propelled Rods, Phys. Rev. Lett. 104, 184502 (2010).

[21] F. Ginelli, The physics of the Vicsek model, Eur. Phys. J. Spec. Top. 225, 2099 (2016).

[22] N. D. Mermin and H. Wagner, Absence of Ferromagnetism or Antiferromagnetism in One- or Two-Dimensional Isotropic Heisenberg Models, Phys. Rev. Lett. 17, 1133 (1966).

[23] J. M. Kosterlitz and D. J. Thouless, Ordering, metastability, and phase transitions in two-dimensional systems, J. Phys. C 6, 1181 (1973).

[24] A. T. Winfree, The Geometry of Biological Time (Springer Science \& Business Media, Berlin, 2001), Vol. 12.

[25] A. Arenas, A. Díaz-Guilera, J. Kurths, Y. Moreno, and C. Zhou, Synchronization in complex networks, Phys. Rep. 469, 93 (2008).

[26] H. Sakaguchi, S. Shinomoto, and Y. Kuramoto, Local and grobal self-entrainments in oscillator lattices, Prog. Theor. Phys. 77, 1005 (1987).

[27] H. Daido, Lower Critical Dimension for Populations of Oscillators with Randomly Distributed Frequencies: A Renormalization-Group Analysis, Phys. Rev. Lett. 61, 231 (1988).

[28] H. Hong, H. Park, and M. Y. Choi, Collective synchronization in spatially extended systems of coupled oscillators with random frequencies, Phys. Rev. E 72, 036217 (2005).

[29] W. R. DiLuzio, L. Turner, M. Mayer, P. Garstecki, D. B. Weibel, H. C. Berg, and G. M. Whitesides, Escherichia coli swim on the right-hand side, Nature (London) 435, 1271 (2005).

[30] E. Lauga, W. R. DiLuzio, G. M. Whitesides, and H. A. Stone, Swimming in circles: Motion of bacteria near solid boundaries, Biophys. J. 90, 400 (2006).

[31] V. B. Shenoy, D. T. Tambe, A. Prasad, and J. A. Theriot, A kinematic description of the trajectories of listeria monocytogenes propelled by actin comet tails, Proc. Natl. Acad. Sci. USA 104, 8229 (2007).

[32] I. H. Riedel, K. Kruse, and J. Howard, A self-organized vortex array of hydrodynamically entrained sperm cells, Science 309, 300 (2005).

[33] Y. Yang, J. Elgeti, and G. Gompper, Cooperation of sperm in two dimensions: Synchronization, attraction, and aggregation through hydrodynamic interactions, Phys. Rev. E 78, 061903 (2008).

[34] Y. Sumino, K. H. Nagai, Y. Shitaka, D. Tanaka, K. Yoshikawa, H. Chaté, and K. Oiwa, Large-scale vortex lattice emerging from collectively moving microtubules, Nature (London) $\mathbf{4 8 3}$, 448 (2012).

[35] K. Kim, N. Yoshinaga, S. Bhattacharyya, H. Nakazawa, M. Umetsu, and W. Teizer, Large-scale chirality in an active layer of microtubules and kinesin motor proteins, Soft Matter 14, 3221 (2018).

[36] M. Loose and T. J. Mitchison, The bacterial cell division proteins FtsA and FtsZ self-organize into dynamic cytoskeletal patterns, Nat. Cell Biol. 16, 38 (2014).
[37] F. Kümmel, B. ten Hagen, R. Wittkowski, I. Buttinoni, R. Eichhorn, G. Volpe, H. Löwen, and C. Bechinger, Circular Motion of Asymmetric Self-Propelling Particles, Phys. Rev. Lett. 110, 198302 (2013).

[38] M. S. D. Wykes, J. Palacci, T. Adachi, L. Ristroph, X. Zhong, M. D. Ward, J. Zhang, and M. J. Shelley, Dynamic selfassembly of microscale rotors and swimmers, Soft Matter $\mathbf{1 2}$ 4584 (2016).

[39] S. van Teeffelen and H. Löwen, Dynamics of a Brownian circle swimmer, Phys. Rev. E 78, 020101(R) (2008).

[40] M. Mijalkov and G. Volpe, Sorting of chiral microswimmers, Soft Matter 9, 6376 (2013).

[41] B. Liebchen, M. E. Cates, and D. Marenduzzo, Pattern formation in chemically interacting active rotors with self-propulsion, Soft Matter 12, 7259 (2016).

[42] H. Löwen, Chirality in microswimmer motion: From circle swimmers to active turbulence, Eur. Phys. J. Spec. Top. 225, 2319 (2016).

[43] F. J. Sevilla, Diffusion of active chiral particles, Phys. Rev. E 94, 062120 (2016).

[44] B. Liebchen and D. Levis, Collective Behavior of Chiral Active Matter: Pattern Formation and Enhanced Flocking, Phys. Rev. Lett. 119, 058002 (2017).

[45] Y. Yang, F. Qiu, and G. Gompper, Self-organized vortices of circling self-propelled particles and curved active flagella, Phys. Rev. E 89, 012720 (2014).

[46] J. Denk, L. Huber, E. Reithmann, and E. Frey, Active Curved Polymers form Vortex Patterns on Membranes, Phys. Rev. Lett. 116, 178301 (2016).

[47] D. Levis and B. Liebchen, Micro-flock patterns and macroclusters in chiral active Brownian disks, J. Phys.: Condens. Matter 30, 084001 (2018).

[48] G.-J. Liao and S. H. L. Klapp, Clustering and phase separation of circle swimmers dispersed in a monolayer, Soft Matter 14, 7873 (2018).

[49] Q.-L. Lei, M. P. Ciamarra, and R. Ni, Non-equilibrium strong hyperuniform fluids of athermal active circle swimmers with giant local fluctuations, Sci. Adv. 5, eaau7423 (2019).

[50] V. Dossetti and F. J. Sevilla, Emergence of Collective Motion in a Model of Interacting Brownian Particles, Phys. Rev. Lett. 115, 058301 (2015).

[51] A. A. Chepizhko and V. L. Kulinskii, On the relation between Vicsek and Kuramoto models of spontaneous synchronization, Phys. A (Amsterdam, Neth.) 389, 5347 (2010).

[52] S. Gupta, A. Campa, and S. Ruffo, Kuramoto model of synchronization: Equilibrium and nonequilibrium aspects, J. Stat. Mech. (2014) R08001.

[53] M. Frasca, A. Buscarino, A. Rizzo, L. Fortuna, and S. Boccaletti, Synchronization of Moving Chaotic Agents, Phys. Rev. Lett. 100, 044102 (2008).

[54] K. Uriu and L. G. Morelli, Collective cell movement promotes synchronization of coupled genetic oscillators, Biophys. J. 107, 514 (2014).

[55] R. Großmann, F. Peruani, and M. Bär, Superdiffusion, largescale synchronization, and topological defects, Phys. Rev. E 93, 040102(R) (2016).

[56] D. Levis, I. Pagonabarraga, and A. Diaz-Guilera, Synchronization in Dynamical Networks of Locally Coupled Self-Propelled Oscillators, Phys. Rev. X 7, 011028 (2017). 
[57] See Supplemental Material at http://link.aps.org/supplemental/ 10.1103/PhysRevResearch.1.023026 for details on the hydrodynamic theory and particle-based simulations.

[58] G. Gübitz and M. G. Schmid, Chiral separation by chromatographic and electromigration techniques, Biopharm. Drug. Dispos. 22, 291 (2001).

[59] C. A. Weber, T. Hanke, J. Deseigne, S. Léonard, O. Dauchot, E. Frey, and H. Chaté, Long-Range Ordering of Vibrated Polar Disks, Phys. Rev. Lett. 110, 208001 (2013).

[60] C. Scholz, M. Engel, and T. Pöschel, Rotating robots move collectively and self-organize, Nat. Commun. 9, 931 (2018).

[61] C. Chen, S. Liu, X.-q. Shi, H. Chaté, and Y. Wu, Weak synchronization and large-scale collective oscillation in dense bacterial suspensions, Nature (London) 542, 210 (2017).

[62] N. Oliver, C. Alpmann, Á. Barroso, L. Dewenter, M. Woerdemann, and C. Denz, Synchronization in pairs of rotating active biomotors, Soft Matter 14, 3073 (2018).

[63] F. Peruani, A. Deutsch, and M. Bär, A mean-field theory for self-propelled particles interacting by velocity alignment mechanisms, Eur. Phys. J. Spec. Top. 157, 111 (2008).

[64] F. D. C. Farrell, M. C. Marchetti, D. Marenduzzo, and J. Tailleur, Pattern Formation in Self-Propelled Particles with Density-Dependent Motility, Phys. Rev. Lett. 108, 248101 (2012).

[65] J. A. Acebrón, L. L. Bonilla, C. J. Pérez-Vicente, F. Ritort, and R. Spigler, The Kuramoto model: A simple paradigm for synchronization phenomena, Rev. Mod. Phys. 77, 137 (2005).

[66] O. Chepizhko and F. Peruani, Diffusion, Subdiffusion, and Trapping of Active Particles in Heterogeneous Media, Phys. Rev. Lett. 111, 160604 (2013).
[67] P. Romanczuk, M. Bär, W. Ebeling, B. Lindner, and L. Schimansky-Geier, Active Brownian particles, Eur. Phys. J. Spec. Top. 202, 1 (2012).

[68] M. E. Cates and J. Tailleur, Motility-induced phase separation, Annu. Rev. Condens. Matter Phys. 6, 219 (2015).

[69] P. Digregorio, D. Levis, A. Suma, G. Gonnella, L. Cugliandolo, and I. Pagonabarraga, Full Phase Diagram of Self-Propelled Hard Disks: From Melting to Motility Induced Phase Separation, Phys. Rev. Lett. 121, 098003 (2018).

[70] D. Tanaka, General Chemotactic Model of Oscillators, Phys. Rev. Lett. 99, 134103 (2007).

[71] S. Sarkar and P. Parmananda, Synchronization of an ensemble of oscillators regulated by their spatial movement, Chaos 20 , 043108 (2010).

[72] N. Kruk, Y. Maistrenko, N. Wenzel, and H. Koeppl, Selfpropelled chimeras, Phys. Rev. E 98, 032219 (2018).

[73] M. Starnini, M. Frasca, and A. Baronchelli, Emergence of metapopulations and echo chambers in mobile agents, Sci. Rep. 6, 31834 (2016).

[74] K. P. O'Keeffe, H. Hong, and S. H. Strogatz, Oscillators that sync and swarm, Nat. Commun. 8, 1504 (2017).

[75] D. S. Dean, Langevin equation for the density of a system of interacting Langevin processes, J. Phys. A. 29, L613 (1996).

[76] E. Bertin, M. Droz, and G. Grégoire, Hydrodynamic equations for self-propelled particles: Microscopic derivation and stability analysis, J. Phys. A 42, 445001 (2009).

[77] A. Peshkov, E. Bertin, F. Ginelli, and H. Chaté, BoltzmannGinzburg-Landau approach for continuous descriptions of generic Vicsek-like models, Eur. Phys. J. Spec. Top. 223, 1315 (2014). 